\title{
Standardization of administered activities in paediatric nuclear medicine: the EANM perspective
}

\author{
Michael Lassmann $^{1} \cdot$ Uta Eberlein $^{1} \cdot$ Egesta Lopci $^{2} \cdot$ Arturo Chiti $^{2,3}$ (D)
}

Received: 21 July 2016 / Accepted: 22 July 2016/Published online: 6 August 2016

(C) Springer-Verlag Berlin Heidelberg 2016

\section{The nuclear medicine global initiative}

In the summer of 2012, the Nuclear Medicine Global Initiative (NMGI) was formed with the intention of promoting and maintaining worldwide standards in the practice and science of nuclear medicine and molecular imaging by encouraging global collaboration in education and by harmonizing procedure guidelines and other policies. The endeavour involved the participation of 13 international organizations with direct involvement in nuclear medicine (Table 1). One of the first projects the NMGI decided to pursue was the standardization of administered activities in paediatric nuclear medicine. As a result, a series of two reports (part 1 and part 2) appeared in the Journal of Nuclear Medicine [1, 2].

Part 1 of the report [1] provides a literature review of the role of paediatric nuclear medicine, the current understanding of the carcinogenic risk of radiation as it pertains to the administration of radiopharmaceuticals in children, and the application of dosimetric models in the paediatric population. It is well understood that children are at higher risk of adverse effects from ionizing radiation than adults, and it is therefore prudent to be more cautious when determining the administered activities for studies in children [1]. This can be challenging because the dosimetric models available for use in

Arturo Chiti

arturo.chiti@hunimed.eu

1 Department of Nuclear Medicine, University Würzburg, Würzburg, Germany

2 Department of Nuclear Medicine, Humanitas Research Hospital, Milano, Italy

3 Humanitas University, Via A. Manzoni 113, 20089 Rozzano, MI, Italy children and adolescents are not as well established as those for use in adults, particularly with respect to the biodistribution of radiopharmaceuticals, as shown in the framework of the European Union (EU) sponsored project PEDDOSE.NET [3, 4]. Part 1 of the report also provides a variety of educational and informational resources regarding the practice, dosimetry, and radiation risk associated with paediatric nuclear medicine. In addition, the report provides a list of links to many resources that are of clear value to the nuclear medicine practitioner.

Part 2 of the report [2] discusses current standards for administered activities in children and adolescents that have been developed by various professional organizations. In addition, an evaluation of the current practice of paediatric nuclear medicine specifically with regard to administered activities, as determined by an international survey of 313 nuclear medicine clinics and centres from 29 countries, is presented. Lastly, the report provides recommendations for a path forward toward global standardization of the administration of radiopharmaceuticals in children.

\section{Survey on the current practice of paediatric nuclear medicine: European point of view}

One of the tasks of the NMGI was to conduct a worldwide survey on the current practice of paediatric nuclear medicine. As described in more detail in part 2 of the NMGI report [2], the survey captured data regarding the top five paediatric imaging procedures, and questions on the technology used and on the activities that would be administered to hypothetical cases at that site. In addition, details on the CT part of hybrid imaging were included.

The survey was open for approximately 1 month (21 July 2014 to 19 August 2014). The results to be reported were 
Table 1 Institutions participating in the Nuclear Medicine Global Initiative

Institution

Asia Oceania Federation of Nuclear Medicine and Biology

Australian and New Zealand Society of Nuclear Medicine

Canadian Association of Nuclear Medicine

Chinese Society of Nuclear Medicine

European Association of Nuclear Medicine

International Atomic Energy Agency

Japanese Society of Nuclear Medicine

Korean Society of Nuclear Medicine

Latin American Association of Societies of Nuclear Medicine and Biology

Society of Nuclear Medicine India

Society of Nuclear Medicine and Molecular Imaging

South African Society of Nuclear Medicine

World Federation on Nuclear Medicine and Biology

from 313 respondents from 29 different countries, although possible inaccuracies might remain in these entries.

Within the member nations of the EANM there were 77 responses. The corresponding distribution is shown in Table 2. This variability in response among countries and regions may limit the accuracy and generalizability of the survey results. Particularly for Europe, the missing or low number of responses from Northern Europe and the UK could have distorted the results. According to the report [2], the five most frequent nuclear medicine procedures in Europe were renograms, bone scans, DMSA scans, MIBG scans, and scans for

Table 2 Participating sites

\begin{tabular}{ll}
\hline Country & $\begin{array}{l}\text { Number of } \\
\text { participating sites }\end{array}$ \\
\hline Albania & 1 \\
Belgium & 7 \\
Croatia & 1 \\
Czech Republic & 7 \\
Denmark & 2 \\
Germany & 4 \\
Hungary & 6 \\
Israel & 11 \\
Italy & 21 \\
Malta & 1 \\
Netherlands & 7 \\
Portugal & 4 \\
Serbia & 2 \\
Spain & 1 \\
Turkey & 2 \\
Total & 77 \\
\hline
\end{tabular}

Meckel's diverticulum. For the eight hypothetical cases $\left({ }^{99 \mathrm{~m}} \mathrm{Tc}\right.$ bone scans, ${ }^{99 \mathrm{~m}} \mathrm{Tc}$ renograms, ${ }^{99 \mathrm{~m}} \mathrm{Tc}$-DMSA scans, and ${ }^{18} \mathrm{~F}$-FDG scans), at the majority of centres the corresponding values of the EANM paediatric dosage card or lower values would be applied [5-7]. There were, however, some outlier centres where the activities applied were twofold or more higher.

With respect to hybrid imaging systems the results are inconclusive [2]. Answers to questions regarding the acquisition of CT in the context of PET/CT were received from 120 of the 313 sites. At $72 \%$ of the sites, automatic exposure control was used for $\mathrm{CT}$ acquisitions. However, $9 \%$ of the respondents did not know if this feature was used at their site, and so the percentage of sites where this feature was used may have been higher than $72 \%$. At about one-third of sites the CT component of PET/CT was acquired in children as a diagnostic scan with administration of contrast agent. A GE PET/CT system was used at about one-third of the sites, a Siemens system at one-third and a Philips system at $20 \%$ [2].

\section{Efforts by the EANM on paediatric dosing}

The first international effort to harmonize the administered activities in paediatric nuclear medicine initiated by the Paediatric Task Group of the European Association of Nuclear Medicine (EANM) was in the late 1980s. The resulting recommendations ("A radiopharmaceuticals schedule for imaging in paediatrics") [8] were published and endorsed by the EANM in 1990. The approach of the authors [8] was to calculate the fraction of the administered activity as a function of the body surface area and to translate the resulting table into a weight-dependent table of fractions of activity to administer. A secondary aim was to provide the minimum amount of administered activity for 24 radiopharmaceuticals, mainly ${ }^{99 \mathrm{~m}} \mathrm{Tc}$-labelled tracers.

In 2005 Jacobs et al. [9] performed a study with the aim firstly to determine if the 1990 version of the EANM dosage card resulted in weight-independent effective doses or weightindependent count rates and secondly to determine whether one dosage card was sufficient for 95 different radiopharmaceuticals, and, if not, how many cards were reasonably needed to take into account intertracer variability. Jacobs et al. calculated normalization factors for count rate and effective doses as a function of body weight. The result of this work was that normalization factors for count rate and effective doses can be estimated accurately as a function of body weight using only one parameter. As a consequence, 95 radiopharmaceuticals could be classified into three clusters A, B and C. Cluster A includes tracers for renal studies, cluster B includes all remaining tracers, except iodine-labelled tracers for thyroid studies which belong to cluster $\mathrm{C}$. The authors therefore suggested 
three tracer-dependent dosage cards to obtain weightindependent effective doses [9].

Based on this work, the EANM paediatrics and dosimetry committees developed and published a new version of the 1990 dosage card for 23 radiopharmaceuticals [5]. The radiopharmaceuticals included in the new dosage card were identified by the EANM committees to be of relevance in paediatric nuclear medicine. In 2008 an amendment to the 2007 EANM dosage card was published [6]. In addition, an online dosage calculator was released by the EANM (http://www.eanm. org/publications/dosage calculator.php?navId=285).

In 2011, the EANM endorsed the PEDDOSE.NET research project of the EU, and an overview of data on dosimetry for the most commonly used diagnostic radiopharmaceuticals was published to provide the basis for subsequent recommendations [3, 4]. This project showed that data on dosimetry were available for many commonly used radiopharmaceuticals, although the data collection and calculation methods were heterogeneous and most of the data had been acquired more than 20 years previously. Moreover, biokinetics and/or absorbed doses for paediatric nuclear medicine applications calculated with clinical data were missing in most cases.

In 2012, as an offshoot of the PEDDOSE.NET project, an application for iPhone/iPad (iAPP) was created to facilitate the calculation of administered activities in paediatric nuclear medicine (http://itunes.apple. com/us/app/peddose/id492680472? $\mathrm{mt}=8$ ). A version for the Android operating system has also been available since 2013 (https://play.google.com/store/apps/details?id= com.netkey.PedDose). Up to May 2016, both applications have been downloaded more than 10,000 times.

In 2014, a joint working group of the EANM and the SNMMI worked on harmonizing the guidelines of the two societies and came up with a joint publication on harmonized values for the activities to be administered in paediatric nuclear medicine [10]. In 2016, corresponding values for ${ }^{68} \mathrm{Ga}$-labelled peptides will be added to the dosage card, based on the recommendations of Machado et al. [11].

\section{Outlook and conclusions}

Based on the results of the NMGI, the work of various groups within the EANM has led to the following observations and conclusions:

- Care must be taken to ensure that paediatric nuclear medicine studies are performed appropriately in those patients who will benefit from the procedure.

- Much information is available both in print and online regarding the appropriate application of nuclear medicine in children, and regarding our current understanding of radiation dosimetry in these patients. Nuclear medicine professionals who image children should take advantage of these materials in order to be better informed and thus to be better able to serve their paediatric patients.

- Although the EANM recommendations for activity dosages in paediatric nuclear medicine have been in place for more than 20 years, there is still variability in the practice of paediatric nuclear medicine throughout Europe. The EANM through its committees is working to understand the reasons for this variability and to suggest possible corrective actions.

- There remain gaps in our knowledge of the biokinetics and radiation dosimetry associated with the application of nuclear medicine in children. There is also limited information regarding the potential risk of adverse health effects from ionizing radiation in children at the dose levels most pertinent to nuclear medicine diagnostics. A more complete understanding of these issues would allow optimization of paediatric nuclear medicine procedures.

As a result of these observations, the EANM endorses the following recommendations:

- The administered activities in paediatric patients should be incorporated into audit processes for nuclear medicine sites, whether by local or country-based programmes, or other audit methods (e.g. the IAEA QUANUM programme).

- Paediatric dose recommendations should be incorporated into a formal training curriculum, and recertification programmes, for all nuclear medicine professionals.

- The appropriate use of paediatric nuclear medicine and the adherence to guidelines for administered activities in children and adolescents should be very actively promoted to a wide audience.

- Further efforts are needed to harmonize dosages in paediatric nuclear medicine procedures worldwide.

\section{Compliance with ethical standards}

Conflicts of interest None.

Ethical approval This article does not describe any studies with human participants or animals performed by any of the authors.

\section{References}

1. Fahey FH, Bom HH, Chiti A, Choi YY, Huang G, Lassmann M, et al. Standardization of administered activities in pediatric nuclear medicine: a report of the first nuclear medicine global initiative project, part 1 - statement of the issue and a review of available resources. J Nucl Med. 2015;56:646-51.

2. Fahey FH, Bom HH, Chiti A, Choi YY, Huang G, Lassmann M, et al. Standardization of administered activities in pediatric nuclear 
medicine: a report of the first nuclear medicine global initiative project, part 2: current standards and the path toward global standardization. J Nucl Med. 2016;57:1148-57.

3. Eberlein U, Broer JH, Vandevoorde C, Santos P, Bardies M, Bacher $\mathrm{K}$, et al. Biokinetics and dosimetry of commonly used radiopharmaceuticals in diagnostic nuclear medicine - a review. Eur J Nucl Med Mol Imaging. 2011;38:2269-81.

4. Eberlein U, Broer JH, Vandevoorde C, Santos P, Lisbona A, Bardies M. PEDDOSE.NET: dosimetry and health effects of diagnostic applications of radiopharmaceuticals with particular emphasis on the use in children and adolescents. Eur J Nucl Med Mol Imaging. 2011;38:S438.

5. Lassmann M, Biassoni L, Monsieurs M, Franzius C, Jacobs F. The new EANM paediatric dosage card. Eur J Nucl Med Mol Imaging. 2007;34:796-8.

6. Lassmann M, Biassoni L, Monsieurs M, Franzius C. The new EANM paediatric dosage card: additional notes with respect to F-18. Eur J Nucl Med Mol Imaging. 2008;35:1666-8.

7. Lassmann M, Treves ST. Pediatric radiopharmaceutical administration: harmonization of the 2007 EANM paediatric dosage card (version 1.5.2008) and the 2010 North American consensus guideline. Eur J Nucl Med Mol Imaging. 2014;41:1636.

8. Piepsz A, Hahn K, Roca I, Ciofetta G, Toth G, Gordon I, et al. A radiopharmaceuticals schedule for imaging in paediatrics. Paediatric Task Group European Association Nuclear Medicine. Eur J Nucl Med. 1990;17:127-9.

9. Jacobs F, Thierens H, Piepsz A, Bacher K, Van de Wiele C, Ham H, et al. Optimised tracer-dependent dosage cards to obtain weightindependent effective doses. Eur J Nucl Med Mol Imaging. 2005;32:581-8.

10. Lassmann M, Treves ST. EANM SNMMI Paediatric Dosage Harmonization Working Group. Paediatric radiopharmaceutical administration: harmonization of the 2007 EANM paediatric dosage card (version 1.5.2008) and the 2010 North American consensus guidelines. Eur J Nucl Med Mol Imaging. 2014;41:1036-41.

11. Machado JS, Beykan S, Herrmann K, Lassmann M. Recommended administered activities for Ga-labelled peptides in paediatric nuclear medicine. Eur J Nucl Med Mol Imaging. 2016. doi:10.1007 /s00259-015-3289-x 